\title{
WEAKLY NONLINEAR IMPULSIVE BOUNDARY VALUE PROBLEMS FOR SYSTEMS OF INTEGRODIFFERENTIAL EQUATIONS
}

\author{
I. BONDAR, M. GROMYAK, AND N. KOZLOVA \\ Received 01 December, 2015
}

\begin{abstract}
Necessary and sufficient conditions for the existence of solutions of weakly nonlinear boundary-value problem of systems of integrodifferential equations with impulsive action at fixed points of time are obtained. Convergent iterative procedure of the solution and establish the connection between the necessary condition and the sufficient condition are presented.
\end{abstract}

2010 Mathematics Subject Classification: 34B15; 34B37

Keywords: integrodifferential equations, weakly nonlinear boundary-value problem, Moore -Penrose pseudoinverse matrix, impulsive action, a generating solution, the equation for generating constants, iterative process.

\section{Statement OF THE PROBLEM AND AUXILIARY RESUlts}

In the mathematical modeling of actual processes with short-term pertubations, it is often possible to neglect the duration of perturbations. These perturbations are regarded as "instantaneous". The indicated idealization leads to the necessity of investigation of dynamical systems with discontinuous trajectories, which are often called dynamical systems with impulsive action. New origional results in the theory of boundary-value problems for differential systems with impulsive action were obtained by using classical methods of the periodic theory of nonlinear oscillations together with methods based on the theory of generalized inverse operators and generalized Green operators $[2-6,11,15]$.

Conditions for the existence of solutions of systems of linear integrodifferential equations and boundary-value problems for them were studied in research papers $[9,10]$, and research papers $[7,8]$ were dedicated to nonlinear occasion systems of integrodifferential equations and boundary-value problems for them, for such kind of problems there was developed general theory effective methods of finding solutions.

The first author was supported in part by Grant of NAS of Ukraine for young scientists (20152016; according to the Agreement No. 1-07-2015 of 01. 07. 2015 and additional agreement No. 1 of 02. 10. 2015). 
In the given research paper by means of Moore-Penrose pseudoinverse matrices and constructive methods nonlinear systems analysis there were investigated conditions for the existence and there were suggested iterative algorithms of constructing solutions of boundary-value problems for weakly nonlinear system of integrodifferential equations with impulsive action.

Consider a weakly nonlinear system of integrodifferential equations with impulsive action at fixed points of time

$$
\begin{gathered}
\dot{x}(t)-\Phi(t) \int_{a}^{b}[A(s) x(s)+B(s) \dot{x}(s)] d s= \\
f(t)+\varepsilon \int_{a}^{b} K(t, s) Z(x(s, \varepsilon), s, \varepsilon) d s, \\
\left.\Delta E_{i} x\right|_{t=\tau_{i}}:=S_{i} x\left(\tau_{i}-0\right)+\gamma_{i}+\varepsilon J_{1}(x(\cdot, \varepsilon), \varepsilon), \\
t \neq \tau_{i}, t \in[a, b], \tau_{i} \in(a, b), i=1,2, \ldots, p,
\end{gathered}
$$

and with boundary condition

$$
\ell x(\cdot)=\alpha+\varepsilon J_{2}(x(\cdot, \varepsilon), \varepsilon), \alpha \in \mathbb{R}^{q}
$$

Here, we use the assumptions and notation from [7, 10, 12]: $A(t), B(t), \Phi(t)$ are, respectively, $(m \times n),(m \times n),(n \times m)$ matrices which components are sought in the space $L_{2}[a, b]$; column vectors of matrice $\Phi(t)$ are linearly independent at $[a, b]$, the $n \times 1$ vector function $f(t) \in L_{2}[a, b] ; \gamma_{i}$ is an $k_{i}$-dimensional column vector of constants, $E_{i}, S_{i}$ are $\left(k_{i} \times n\right)$ constant matrices such that $\operatorname{rank}\left(E_{i}+S_{i}\right)=k_{i}(i=$ $1,2, \ldots, p)$, i. e., the solution of the system is determined by the unique extension through the point of discontinuity

$$
\left.\Delta E_{i} x\right|_{t=\tau_{i}}=E_{i}\left(x\left(\tau_{i}+0\right)-x\left(\tau_{i}-0\right)\right),
$$

$\alpha=\operatorname{col}\left(\alpha_{1}, \alpha_{2}, \alpha_{3}, \ldots, \alpha_{p}\right) \in \mathbb{R}^{p}, \ell-$ is a bounded linear $p$-dimensional vector functional, $D_{2}[a ; b], \ell=\operatorname{col}\left(\ell_{1}, \ell_{2}, \ell_{3}, \ldots, \ell_{p}\right): D_{2}[a ; b] \rightarrow \mathbb{R}^{p} ; Z(x(t, \varepsilon), t, \varepsilon)-$ is an $n$-dimensional vector function nonlinear with respect to the first component, continuously differentiable with respect to $x$ in the vicinity of a generating solution, integrable with respect to $t$; and continuous in $\varepsilon$ :

$$
Z(\cdot, t, \varepsilon) \in C^{1}\left[\left\|x-x_{0}\right\| \leq \mu\right], Z(x(\cdot, \varepsilon), \cdot, \varepsilon) \in L_{2}[a, b], Z(x(t, \cdot), t, \cdot) \in C\left[0, \varepsilon_{0}\right] ;
$$

$J_{1}(x(\cdot, \varepsilon), \varepsilon), J_{2}(x(\cdot, \varepsilon), \varepsilon)$ are, respectively, nonlinear bounded

$p, q$-dimensional vector functionals continuously differentiable with respect to $x$ in the Frechet sense and continuous in $\varepsilon$ in the vicinity of a generating solution.

We seek the solution of problem (1.1)-(1.3) in the class of vector functions $x(t)=$ $x(t,, \varepsilon)$ such that

$$
\left.x(\cdot, \varepsilon) \in D_{2}[a, b] \backslash\left\{\tau_{i}\right\}_{I}\right), \dot{x}(\cdot, \varepsilon) \in L_{2}[a, b], x(t, \cdot) \in C\left[0, \varepsilon_{0}\right] .
$$


The norms in the spaces $D_{2}\left([a, b] \backslash\left\{\tau_{i}\right\}_{I}\right)$ and $L_{2}[a, b], C\left[0, \varepsilon_{0}\right]$ are introduced in the standart way (by analogy with $[1,6]$ ).

We show that the problem with impulsive action (1.1)- (1.2) can be investigated if we consider this problem as an "interface" boundary-value problem [17]. To reveal this relationship, we introduce a $k$-dimensional linear bounded vector functional $\varphi x(\cdot)$ :

$$
\begin{gathered}
\varphi:=\operatorname{col}\left(\varphi_{1}, \ldots, \varphi_{p}\right): D_{2}\left([a, b] \backslash\left\{\tau_{i}\right\}_{I}\right) \rightarrow \mathbb{R}^{k}, k:=k_{1}+k_{2}+\ldots+k_{p}, \\
\left.\varphi_{i}: D_{2}[a, b] \backslash\left\{\tau_{i}\right\}_{I}\right) \rightarrow \mathbb{R}^{k_{i}},(i=1, \ldots, p)
\end{gathered}
$$

as follows:

$$
\left\{\begin{array}{l}
\varphi_{1} x:=E_{1} x\left(\tau_{1}+\right)-\left(E_{1}+S_{1}\right) x\left(\tau_{1}-\right) \\
\varphi_{2} x:=E_{2} x\left(\tau_{2}+\right)-\left(E_{2}+S_{2}\right) x\left(\tau_{2}-\right) \\
\cdots \ldots \ldots \ldots \ldots \ldots \ldots \ldots \ldots \ldots \ldots \ldots \ldots \ldots \\
\varphi_{p} x:=E_{p} x\left(\tau_{p}+\right)-\left(E_{p}+S_{p}\right) x\left(\tau_{p}-\right)
\end{array}\right.
$$

and represent the impulsive action (1.2) as the boundary condition

$$
\varphi x(\cdot)=\gamma+\varepsilon J_{1}(x(\cdot, \varepsilon), \varepsilon),
$$

where $\gamma=\operatorname{col}\left(\gamma_{1}, \gamma_{2}, \ldots, \gamma_{p}\right) \in \mathbb{R}^{k}, \gamma_{i} \in \mathbb{R}^{k_{i}}$.

We introduce the bounded linear $(k+q)$-dimensional vector functional

$$
\left.\mathfrak{L}:=\left[\begin{array}{c}
\varphi \\
\ell
\end{array}\right]: D_{2}[a, b] \backslash\left\{\tau_{i}\right\}_{I}\right) \rightarrow \mathbb{R}^{k+q}
$$

and write the impulse condition (1.6) with boundary condition (1.3) in the next form

$$
\mathfrak{L} x(\cdot)=\delta+\varepsilon J(x(\cdot, \varepsilon), \varepsilon) \in R^{k+q},
$$

where $\delta:=\left[\begin{array}{l}\gamma \\ \alpha\end{array}\right], \delta \in R^{k+q}, J\left(x(\cdot, \varepsilon):=\left[\begin{array}{l}J_{1}(x(\cdot, \varepsilon) \\ J_{2}(x(\cdot, \varepsilon)\end{array}\right]\right.$ - is a nonlinear bounded $k+q$ dimensional vector functional continuously differentiable with respect to $x$ in the Frechet sense and continuous in $\varepsilon$ in the vicinity of a generating solution.

Now the impulse weakly nonlinear boundary-value problem (1.1)-(1.3) we can consider as weakly nonlinear boundary-value problem (1.1), (1.7). Similarly, as in the [7], we can establish the necessary and sufficient conditions of solvability and the relationship between them for so obtained weakly nonlinear boundary value problem for integro-differential equations (1.1), (1.7).

So, we looking a solution $x=x(t, \varepsilon)$ of boundary-value problem (1.1),(1.7) which is defined in this class of vector functions

$$
\begin{gathered}
x=x(t): x(\cdot) \in D_{2}\left([a, b] \backslash\left\{\tau_{i}\right\}_{I}\right), \dot{x}(\cdot) \in L_{2}[a, b] . \\
\left.x(\cdot, \varepsilon) \in D_{2}[a, b] \backslash\left\{\tau_{i}\right\}_{I}\right), \dot{x}(\cdot, \varepsilon) \in L_{2}[a, b], x(t, \cdot) \in C\left[0, \varepsilon_{0}\right],
\end{gathered}
$$


and which for $\varepsilon=0$ converted to one with solutions generating boundary-value problem

$$
\begin{gathered}
\dot{x}(t)-\Phi(t) \int_{a}^{b}[A(s) x(s)+B(s) \dot{x}(s)] d s=f(t), \\
\mathfrak{L} x(\cdot)=\delta .
\end{gathered}
$$

We now present the following known criterion of solvability for the generating boundary-value problem (1.8), (1.9):

Theorem 1 ([16]). Let $\operatorname{rank} Q \leq\left(k+q, r_{1}\right)$. Then the homogeneous boundaryvalue problem (1.8), (1.9) $(f(t)=0, \delta=0)$ has linearly independent solutions of the form:

$$
\begin{aligned}
& x\left(t, c_{r}\right)=\Psi_{0}(t) P_{D_{r_{1}}} P_{Q_{r}} c_{r}, c_{r} \in \mathbb{R}^{r}, \\
& r_{1}=m+n-\operatorname{rank} D, r=r_{1}-\operatorname{rank} Q .
\end{aligned}
$$

The inhomogeneous boundary-value problem (1.8), (1.9) is solvable if and only if the conditions:

$$
\begin{gathered}
P_{D_{d_{1}}^{*}} \tilde{b}=0, \quad P_{Q_{d_{2}}^{*}}(\delta-\mathfrak{L} F(\cdot))=0, \\
d_{1}=m-\operatorname{rank} D, d_{2}=k+q-\operatorname{rank} Q
\end{gathered}
$$

are satisfied. Moreover, it has an $r$-parameter family of linearly independent solutions:

$$
x\left(t, c_{r}\right)=\Psi_{0}(t) P_{D_{r_{1}}} P_{Q_{r}} c_{r}+\Psi_{0}(t) P_{D_{r_{1}}} Q^{+}(\delta-\mathfrak{L} F(\cdot))+F(t), \quad \forall c_{r} \in \mathbb{R}^{r} .
$$

Here, $\tilde{f}(t)=\int_{a}^{t} f(s) d s, \tilde{b}=\int_{a}^{b}[A(s) \tilde{f}(s)+B(s) f(s)] d s$,

$F(t)=\tilde{f}(t)+\Psi_{0}(t) D^{+} \tilde{b}, \Psi_{0}(t)=\left[\Psi(t), I_{n}\right], \Psi(t)=\int_{a}^{t} \Phi(s) d s$

are $n \times(m+n)$ and $n \times m$ matrices, respectively; $D=\left[I_{m}-\int_{a}^{b}[A(s) \Psi(s)+\right.$ $\left.B(s) \Phi(s)] d s,-\int_{a}^{b} A(s) d s\right]$ is an $m \times(m+n)$ matrix, $I_{m}$ and $I_{n}$ are the identity matrices of the corresponding orders; $P_{D}$ and $P_{D^{*}}$ are, respectively, $(m+n) \times(m+$ $n), m \times m$ matrices (orthoprojectors onto the kernel and cokernel of the matrix $D$; $P_{D_{r_{1}}}\left(P_{D_{d_{1}}^{*}}\right)$ is the matrix formed by the complete system of $r_{1}\left(d_{1}\right)$ linearly independent columns (rows) of the matrix (orthoprojector) $P_{D}\left(P_{D^{*}}\right)$. The matrix $Q$ is a $(k+q) \times r_{1}$ matrix constructed according to [6]; $D^{+}\left(Q^{+}\right)$is the Moore-Penrose pseudoinverse matrix for the matrix $D(Q)$. The quantities $P_{Q}$ and $P_{Q^{*}}$ are, respectively, $r_{1} \times r_{1}$ and $(k+q) \times(k+q)$ matrices (orthoprojectors onto the kernel 
and cokernel of the matrix $Q)$ and $P_{Q_{r}}\left(P_{Q_{d_{2}}^{*}}\right)$ is the matrix formed by the complete system of $r\left(d_{2}\right)$ linearly independent columns (rows) of the matrix $P_{Q}\left(P_{Q^{*}}\right)$.

Further, the solution $x(t, 0)=x_{0}\left(t, c_{r}\right)(1.11)$ of generating boundary-value problem (1.8),(1.9) will be call a generating solution of the boundary-value problem for the weakly nonlinear impulsive system of integrodifferential equations (1.1)-(1.3), where $c_{r} \in \mathbb{R}^{r}$ is unknown vector of constants and which will defined below.

\section{Results}

Consider the critical case where the corresponding homogeneous generating $(f(t)=0, \delta=0)$ boundary-value problem (1.8),(1.9) has nontrivial solutions $x\left(t, c_{r}\right)=x_{0}\left(t, c_{r}\right)$, and determined by the formula (1.11).

\subsection{The necessary condition.}

First, we establish the necessary condition of solvability of the boundary-value problem (1.1), (1.7). The following statement is true:

Theorem 2. Assume that the weakly nonlinear boundary-value problem (1.1)(1.3) has a solution $x=x(t, \varepsilon)$ :

$$
\left.x(\cdot, \varepsilon) \in D_{2}[a, b] \backslash\left\{\tau_{i}\right\}_{I}\right), \dot{x}(\cdot, \varepsilon) \in L_{2}[a, b], x(t, \cdot) \in C\left(0, \varepsilon_{0}\right]
$$

which turns, for $\varepsilon=0$ into a generating solution $x_{0}\left(t, c_{r}\right)(1.11)$ with constant $c_{r}=$ $c_{r}^{0}(r=m+n-\operatorname{rank} D-\operatorname{rank} Q)$.

Then the vector of constants $c_{r}^{0}$ is necessarily a real root of the system of equations

$$
\begin{gathered}
P_{D_{d_{1}}^{*}} \int_{a}^{b}\left[A(s) \int_{a}^{s} \int_{a}^{b} K(\tau, s) Z\left(x_{0}\left(s, c_{r}^{0}\right), s, 0\right) d s d \tau+\right. \\
\left.B(s) \int_{a}^{b} K(s, \tau) Z\left(x_{0}\left(\tau, c_{r}^{0}\right), \tau, 0\right) d \tau\right] d s=0, \\
P_{Q_{d_{2}}^{*}}\left\{J\left(x_{0}\left(\cdot, c_{r}^{0}\right), 0\right)-\mathfrak{L}\left(\int_{a}^{b} \int_{a}^{b} K(\tau, s) Z\left(x_{0}\left(s, c_{r}^{0}\right), s, 0\right) d s d \tau+\right.\right. \\
\Psi_{0}(\cdot) D^{+} \int_{a}^{b}\left[A(t) \int_{a}^{t} \int_{a}^{b} K(\tau, s) Z\left(x_{0}\left(s, c_{r}^{0}\right), s, 0\right) d s d \tau+\right. \\
\left.\left.\left.B(t) \int_{a}^{b} K(t, s) Z\left(x_{0}\left(s, c_{r}^{0}\right), s, 0\right) d s\right] d t\right)\right\}=0,
\end{gathered}
$$




$$
d_{1}=m-\operatorname{rank} D, d_{2}=k+q-\operatorname{rank} Q .
$$

The proof is similar to the proofs of Theorem 5.4 in [6] and Theorem 2 in [7]. For periodic problems, the constant $c_{r}^{0}$ has a physical meaning and plays the role of amplitude of the generating solution. Therefore, in the classical periodic problem, the corresponding equation for the system of ordinary differential equations is called the equation for generating amplitudes [14]. Similarly, we say that Eq. (2.1), (2.2) is the equation for generating constants of the boundary-value problem for the system of integrodifferential equations (1.1)-(1.3).

If Eq. (2.1), (2.2) is solvable, then the vector $c_{r}=c_{r}^{0} \in \mathbb{R}^{r}$ specifies the generating solution $x\left(t, c_{r}\right)=x_{0}\left(t, c_{r}^{0}\right)(1.11)$, which may correspond to the solution $x(t, \varepsilon)$ of the original boundary-value problem (1.1)-(1.3) for $\varepsilon=0$. If Eq. (2.1), (2.1) has no solutions, then the boundary-value problem (1.1)-(1.3) also does not have the required solution. Here, we speak about the real roots of the equation for generating constants (2.1), (2.2). Thus, the necessary condition for the solvability of the boundary-value problem (1.1)-(1.3) is satisfied if Eq. (2.1), (2.2) has at least one real solution

$c_{r}=c_{r}^{0} \in R^{r}$.

\subsection{The sufficient condition.}

To establish a sufficient condition for the existence of a solution of the boundaryvalue problem (1.1), (1.7) we perform the change the variables:

$$
x(t, \varepsilon)=x_{0}\left(t, c_{r}^{0}\right)+y(t, \varepsilon) .
$$

In the new variables, we seek the conditions of existence for the solution $y(t, \varepsilon)$ :

$$
y(\cdot, \varepsilon) \in D_{2}\left([a, b] \backslash\left\{\tau_{i}\right\}_{I}\right), \dot{y}(\cdot, \varepsilon) \in L_{2}[a, b], y(t, \cdot) \in C\left[0, \varepsilon_{0}\right], y(t, 0)=0,
$$

which turns, for $\varepsilon=0$ into the trivial solution of the boundary-value problem

$$
\begin{gathered}
\dot{y}(t)-\Phi(t) \int_{a}^{b}[A(s) y(s)+B(s) \dot{y}(s)] d s= \\
\qquad \int_{a}^{b} K(t, s) Z\left(x_{0}\left(s, c_{r}^{0}\right)+y(s, \varepsilon), s, \varepsilon\right) d s, \\
\mathfrak{L} y(\cdot)=\varepsilon J\left(x_{0}\left(\cdot, c_{r}^{0}\right)+y(\cdot, \varepsilon), \varepsilon\right) .
\end{gathered}
$$

In view of the continuous differentiability of the vector function $Z(x, t, \varepsilon)$ and the Frechet differentiability of the vector functional $J(x(\cdot, \varepsilon), \varepsilon)$ with respect to the first components in the vicinity of the point $\varepsilon=0$, we select, in the vector function $Z\left(x_{0}+\right.$ $y, t, \varepsilon)$ and in the vector functional $J\left(x_{0}\left(\cdot, c_{r}^{0}\right)+y(\cdot, \varepsilon), \varepsilon\right)$ their linear parts with 
respect to $y$ and the zero-order terms with respect to $\varepsilon$. As a result, we arrive at the following decomposition:

$$
\begin{aligned}
& Z\left(x_{0}+y, t, \varepsilon\right)=Z\left(x_{0}\left(t, c_{r}^{0}\right), t, 0\right)+A_{1}(t) y(t, \varepsilon)+R(y(t, \varepsilon), t, \varepsilon), \\
& J\left(x_{0}\left(\cdot, c_{r}^{0}\right)+y(\cdot, \varepsilon), \varepsilon\right)=J\left(x_{0}\left(\cdot, c_{r}^{0}\right)\right)+\mathfrak{L}_{1} y(\cdot, \varepsilon)+R_{1}(y(\cdot, \varepsilon), \varepsilon),
\end{aligned}
$$

where

$$
\begin{gathered}
Z\left(x_{0}\left(t, c_{r}^{0}\right), t, 0\right) \in C[a, b], \quad J\left(x_{0}\left(\cdot, c_{r}^{0}\right)\right)=J\left(x_{0}\left(\cdot, c_{r}^{0}\right), 0\right), \\
A_{1}(t)=A_{1}\left(t, c_{r}^{0}\right)=\left.\frac{\partial Z(x, t, 0)}{\partial x}\right|_{x=x_{0}\left(t, c_{r}^{0}\right)} \in C[a, b],
\end{gathered}
$$

and $\mathfrak{L}_{1} y(\cdot, \varepsilon)$ is the linear part of the vector functional $J\left(x_{0}\left(\cdot, c_{r}^{0}\right)+y(\cdot, \varepsilon), \varepsilon\right)$.

The linear operator $\mathfrak{L}_{1}=J^{\prime}\left(x_{0}\right)$ is the Frechet derivative of the vector functiona $J(x(\cdot, \varepsilon), \varepsilon)$ at the point $x=x_{0}\left(t, c_{r}^{0}\right)$. The nonlinear vector function $R(y(t, \varepsilon), t, \varepsilon)$ belongs to the class $C^{1}(\|y\| \leq q), L_{2}[a, b], C\left[0, \varepsilon_{0}\right]$. In this case, we have

$$
R(0, t, 0)=0, \quad \frac{\partial R(0, t, 0)}{\partial y}=0, \quad R_{1}(0,0)=0, \frac{\partial R_{1}(0,0)}{\partial y}=0 .
$$

Taking into account the decomposition of nonlinearities (2.5) and (2.6) in the boundary-value problem (2.3), (2.4), we obtain the boundary-value problem

$$
\begin{aligned}
& \dot{y}(t)-\Phi(t) \int_{a}^{b}[A(s) y(s)+B(s) \dot{y}(s)] d s=p(t, \varepsilon), \\
& \mathfrak{L} y(\cdot)=\varepsilon\left\{J\left(x_{0}\left(\cdot, c_{r}^{0}\right)\right)+\mathfrak{L}_{1} y(\cdot, \varepsilon)+R_{1}(y(\cdot, \varepsilon), \varepsilon)\right\},
\end{aligned}
$$

and seek its solution in the form

$$
y(t, \varepsilon)=X_{r}(t) c+\bar{y}(t, \varepsilon), c=c(\varepsilon) \in \mathbb{R}^{r},
$$

where $X_{r}(t)=\Psi_{0}(t) P_{D_{r_{1}}} P_{Q_{r}}$ ia an $n \times r$ matrix, $c \in \mathbb{R}^{r}$ is an unknown constant determined in what follows and

$$
\begin{aligned}
\bar{y}(t, \varepsilon)=\varepsilon \Psi_{0}(t) P_{D_{r_{1}}} Q^{+}\left\{J\left(x_{0}\left(\cdot, c_{r}^{0}\right)\right)+\mathfrak{L}_{1} y(\cdot, \varepsilon)+\right. \\
\left.\quad R_{1}(y(\cdot, \varepsilon), \varepsilon)-\mathfrak{L} F_{0}(\cdot, \varepsilon)\right\}+F_{0}(t, \varepsilon)
\end{aligned}
$$

By Theorem 1, the inhomogeneous boundary-value problem (2.7), (2.8) is solvable if and only if the conditions

$$
\begin{aligned}
& P_{D_{d_{1}}^{*}} \tilde{b}_{0}(\varepsilon)=0 \\
& P_{Q_{d_{2}}^{*}}\left\{\varepsilon J\left(x_{0}\left(\cdot, c_{r}^{0}\right)+y(\cdot, \varepsilon), \varepsilon\right)-\mathfrak{L} F_{0}(\cdot, \varepsilon)\right\}=0 .
\end{aligned}
$$


Here, $\tilde{b}_{0}(\varepsilon)=\int_{a}^{b}[A(s) \tilde{p}(s, \varepsilon)+B(s) p(s, \varepsilon)] d s$ is an $n \times 1$ column vector whose components belong to the space $C\left[0, \varepsilon_{0}\right]$,

$$
\begin{gathered}
h(t, \varepsilon)=\varepsilon \int_{a}^{b} K(t, s)\left[Z\left(x_{0}\left(s, c_{r}^{0}\right), s, 0\right)+A_{1}(s) y(s, \varepsilon)+\right. \\
R(y(s, \varepsilon), s, \varepsilon)] d s=\varepsilon\left(h_{1}(t)+h_{2}^{0}(t, \varepsilon)\right), \\
h_{1}(t)=\int_{a}^{b} K(t, s) A_{1}(s) X_{r}(s) d s, \\
h_{2}^{0}(t, \varepsilon)=\int_{a}^{b} K(t, s)\left[A_{1}(s) \bar{y}(s, \varepsilon)+R(y(s, \varepsilon), s, \varepsilon)\right] d s, \\
F_{0}(t, \varepsilon)=\tilde{h}(t, \varepsilon)+\Psi_{0}(t) D^{+} \tilde{b}_{0}(\varepsilon)=F_{0}^{1}(t)+F_{0}^{2}(t, \varepsilon), \\
F_{0}^{1}(t)=\tilde{h}_{1}(t)+\Psi_{0}(t) D^{+} \int_{a}^{b}\left[A(s) \tilde{h}_{1}(s)+B(s) h_{1}(s)\right] d s, \\
F_{0}^{2}(t, \varepsilon)=\tilde{h}_{2}^{0}(t, \varepsilon)+\Psi_{0}(t) D^{+} \int_{a}^{b}\left[A(s) \tilde{h}_{2}^{0}(s, \varepsilon)+B(s) h_{2}^{0}(s, \varepsilon)\right] d s, \\
\tilde{h}_{a}^{t} \int_{a}^{t} \int_{a}^{t} h_{1}(s) d s, \tilde{h}_{2}^{0}(t, \varepsilon)=\int_{a}^{0}(s, \varepsilon) d s .
\end{gathered}
$$

In view of the facts that $y(t, \varepsilon)=X_{r}(t) c+\bar{y}(t, \varepsilon)$ and conditions (2.1), (2.2) are satisfied, we obtain the following system for the unknown vector of constants $c \in \mathbb{R}^{r}$ from (2.9) and (2.10)

$$
\begin{aligned}
P_{D_{d_{1}}^{*}}\left\{\int_{a}^{b}\left[A(s) \tilde{h}_{1}(s)+B(s) h_{1}(s)\right] d s\right\} c & = \\
& -P_{D_{d_{1}}^{*}} \int_{a}^{b}\left[A(s) \tilde{h}_{2}^{0}(s, \varepsilon)+B(s) h_{2}^{0}(s, \varepsilon)\right] d s,
\end{aligned}
$$




$$
\begin{aligned}
P_{Q_{d_{2}}^{*}}\left\{\mathfrak{L}_{1} X_{r}(\cdot)-\mathfrak{L} F_{0}^{1}(\cdot)\right\} c= \\
\quad-P_{Q_{d_{2}}^{*}}\left\{J\left(x_{0}\left(\cdot, c_{r}^{0}\right)\right)+\mathfrak{L}_{1} \bar{y}(\cdot, \varepsilon)+R_{1}(y(\cdot, \varepsilon), \cdot, \varepsilon)-\mathfrak{L} F_{0}^{2}(\cdot, \varepsilon)\right\} .
\end{aligned}
$$

System (2.11), (2.12) can be rewritten as follows:

$$
B_{0} c=g \text {, }
$$

with the $\left(d_{1}+d_{2}\right) \times r$ matrix

$$
B_{0}:=\left[\begin{array}{c}
P_{D_{d_{1}}^{*}} \int_{a}^{b}\left[A(s) \tilde{h}_{1}(s)+B(s) h_{1}(s)\right] d s \\
P_{Q_{d_{2}}^{*}}\left\{\mathfrak{L}_{1} X_{r}(\cdot)-\mathfrak{L} F_{0}^{1}(\cdot)\right\}
\end{array}\right],
$$

and the $\left(d_{1}+d_{2}\right) \times 1$ vector function

$$
g:=\left[\begin{array}{c}
-P_{D_{d_{1}}^{*}} \int_{a}^{b}\left[A(s) \tilde{h}_{2}^{0}(s, \varepsilon)+B(s) p_{2}^{0}(s, \varepsilon)\right] d s \\
-P_{Q_{d_{2}}^{*}}\left\{J\left(x_{0}\left(\cdot, c_{r}^{0}\right)\right)+\mathfrak{L}_{1} \bar{y}(\cdot, \varepsilon)+R_{1}(y(\cdot, \varepsilon), \cdot, \varepsilon)-\mathfrak{L} F_{0}^{2}(\cdot, \varepsilon)\right\}
\end{array}\right]
$$

System (2.13) is solvable if and only if the condition

$$
P_{B_{0}^{*}} g=0
$$

is satisfied. Since the vector function $g$ contains unknown quantities, in order to use this condition, we assume, instead of (2.14), that the condition $P_{B_{0}^{*}}=0$ is satisfied, which is equivalent to the condition [6] :

$$
\operatorname{rank} B_{0}=d_{1}+d_{2} \text {. }
$$

Here, $P_{B_{0}^{*}}$ is a $\left(d_{1}+d_{2}\right) \times\left(d_{1}+d_{2}\right)$ matrix (orthoprojector) that projects the spacec $R^{d_{1}+d_{2}}$ onto the null space $N\left(B_{0}^{*}\right)$.

As a result of the solution of system (2.13), we arrive at the equivalent operator system

$$
\begin{gathered}
y(t, \varepsilon)=X_{r}(t) c+\bar{y}(t, \varepsilon), \\
c=-B_{0}^{+} g \\
\bar{y}(t, \varepsilon)=\varepsilon \Psi_{0}(t) P_{D_{r_{1}}} Q^{+}\left\{J\left(x_{0}\left(\cdot, c_{r}^{0}\right)\right)+\mathfrak{L}_{1} y(\cdot, \varepsilon)+\right. \\
\left.+R_{1}(y(\cdot, \varepsilon), \varepsilon)-\mathfrak{L} F_{0}(\cdot, \varepsilon)\right\}+F_{0}(t, \varepsilon) .
\end{gathered}
$$

We introduce $u=\operatorname{col}(y(t, \varepsilon), c(\varepsilon), \bar{y}(t, \varepsilon))$ and rewrite system (2.16) in the new variables as follows:

$$
u=L^{(1)} u+\hat{F} u
$$


where

$$
\begin{aligned}
& L^{(1)}=\left(\begin{array}{ccc}
0 & X_{r}(t) & I_{n} \\
0 & 0 & L_{1} \\
0 & 0 & 0
\end{array}\right), \\
& L_{1} \varphi=-B_{0}^{+}\left[\begin{array}{c}
-P_{D_{d_{1}}^{*}} \int_{a}^{b}\left[A(s) \int_{a}^{s} \int_{a}^{b} K(t, \tau) A_{1}(\tau) \varphi(\tau) d \tau d t+\right. \\
\left.+B(s) \int_{a}^{b} K(s, \tau) A_{1}(\tau) \varphi(\tau) d \tau\right] d s \\
-P_{Q_{d_{2}}^{*}}\left\{\mathfrak{L}_{1} \varphi(\cdot)-\mathfrak{L}\left(\int_{a} \int_{a}^{b} K(\cdot, \tau) A_{1}(\tau) \varphi(\tau) d \tau+\right.\right. \\
+\Psi_{0}(\cdot) D^{+} \int_{a}^{b}\left[A(s) \int_{a}^{b} \int_{a}^{b} K(t, \tau) A_{1}(\tau) \varphi(\tau) d \tau d t+\right. \\
\left.\left.\left.+B(s) \int_{a}^{b} K(s, \tau) A_{1}(\tau) \varphi(\tau) d \tau\right] d s\right)\right\}
\end{array}\right], \\
& \hat{F} u=\left(\begin{array}{c}
0 \\
-B_{0}^{+} \bar{g} \\
\varepsilon \Psi_{0}(t) P_{D_{r_{1}}} Q^{+}\left\{J\left(x_{0}\left(\cdot, c_{r}^{0}\right)\right)+\right. \\
\left.+\mathfrak{L}_{1} y(\cdot, \varepsilon)+R_{1}(y(\cdot, \varepsilon), \varepsilon)-\mathfrak{L} F_{0}(\cdot, \varepsilon)\right\}+F_{0}(t, \varepsilon)
\end{array}\right), \\
& \bar{g}:=\left[\begin{array}{c}
-P_{D_{d_{1}}} \int_{a}^{b}\left[A(s) \int_{a}^{s} \int_{a}^{b} K(t, \tau) R(y(\tau, \varepsilon), \tau, \varepsilon) d \tau d t+\right. \\
\left.B(s) \int_{a}^{b} K(s, \tau) R(y(\tau, \varepsilon), \tau, \varepsilon) d \tau\right] d s \\
-P_{Q_{d_{2}}^{*}}\left\{R_{1}(y(\cdot, \varepsilon), \cdot, \varepsilon)-\mathfrak{L}\left(\int_{a}^{b} \int_{a}^{b} K(\cdot, \tau) R(y(\tau, \varepsilon), \tau, \varepsilon) d \tau+\right.\right. \\
\Psi_{0}(\cdot) D^{+} \int_{a}^{b}\left[A(s) \int_{a}^{s} \int_{a}^{b} K(t, \tau) R(y(\tau, \varepsilon), \tau, \varepsilon) d \tau d t+\right. \\
\left.\left.\left.B(s) \int_{a}^{b} K(s, \tau) R(y(\tau, \varepsilon), \tau, \varepsilon) d \tau\right] d s\right)\right\}
\end{array}\right] .
\end{aligned}
$$

System (2.16) can be rewritten in the form

$$
\left(I_{\varrho}-L^{(1)}\right) u=\hat{F} u, \varrho=n+r+1 .
$$


Since the block-diagonal matrix operator $\left(I_{\varrho}-L^{(1)}\right)$ is always invertible, we can rewrite system (2.16) as follows:

$$
u=S u, S:=\left(I_{\varrho}-L^{(1)}\right)^{-1} \hat{F} .
$$

By the choice of $\varepsilon$ and the neighborhood of the generating solution, in view of the structure of the operator $\hat{F}$, as in $[6,7]$, we can show that $S$ is a contraction operator [13] acting from the space $D_{2}\left([a, b] ; \mathbb{R}^{n}\right) \times C\left(\left[0, \varepsilon_{0}\right] ; \mathbb{R}\right) \times D_{2}\left([a, b] ; \mathbb{R}^{n}\right)$ into itself with the corresponding norm. Hence, the operator equation $u=S u$ has a unique solution, which can be found as follows:

$$
u=\lim _{v \rightarrow \infty} u_{v}, u_{v}=S u_{v-1}, \text { where } u_{0}=\operatorname{col}\left(y_{0}, c_{0}, \bar{y}_{0}\right)=0 .
$$

Returning to the original boundary-value problem (1.1), (1.7), we get the following iterative process for finding the solution.

In the first stage of the iterative process, we obtain the boundary-value problem

$$
\begin{gathered}
\dot{y}_{1}(t)-\Phi(t) \int_{a}^{b}\left[A(s) y_{1}(s)+B(s) \dot{y}_{1}(s)\right] d s= \\
\varepsilon \int_{a}^{b} K(t, s) Z\left(x_{0}\left(s, c_{r}^{0}\right), s, 0\right) d s, \\
\mathfrak{L} y_{1}(\cdot)=\varepsilon J\left(x_{0}\left(\cdot, c_{r}^{0}\right), 0\right) .
\end{gathered}
$$

This problem is solvable if and only if the following conditions are satisfied:

$$
\begin{gathered}
P_{D_{d_{1}}^{*}} \int_{a}^{b}\left[A(s) \int_{a}^{s} \int_{a}^{b} K(\tau, s) Z\left(x_{0}\left(s, c_{r}^{0}\right), s, 0\right) d s d \tau+\right. \\
\left.B(s) \int_{a}^{b} K(s, \tau) Z\left(x_{0}\left(\tau, c_{r}^{0}\right), \tau, 0\right) d \tau\right] d s=0, \\
P_{Q_{d_{2}}^{*}}\left\{J\left(x_{0}\left(\cdot, c_{r}^{0}\right), 0\right)-\mathfrak{L}\left(\int_{a}^{j} \int_{a}^{b} K(\tau, s) Z\left(x_{0}\left(s, c_{r}^{0}\right), s, 0\right) d s d \tau+\right.\right. \\
\Psi_{0}(\cdot) D^{+} \int_{a}^{b}\left[A(t) \int_{a}^{t} \int_{a}^{b} K(\tau, s) Z\left(x_{0}\left(s, c_{r}^{0}\right), s, 0\right) d s d \tau+\right.
\end{gathered}
$$




$$
\left.\left.\left.B(t) \int_{a}^{b} K(t, s) Z\left(x_{0}\left(s, c_{r}^{0}\right), s, 0\right) d s\right] d t\right)\right\}=0 .
$$

These conditions are satisfied because the generating solution satisfies conditions (2.1) and (2.2) in view of the choice of $c_{r}^{0} \in \mathbb{R}^{r}$. Assume that the first approximation $y_{1}(t, \varepsilon)$ to the required solution $y(t, \varepsilon)$ of the boundary-value problem $(2.3),(2.4)$ is equal to $\bar{y}_{1}(t, \varepsilon)$. This yields

$$
y_{1}(t, \varepsilon)=\bar{y}_{1}(t, \varepsilon)=\varepsilon \Psi_{0}(t) P_{D_{r_{1}}} Q^{+}\left\{J\left(x_{0}\left(\cdot, c_{r}^{0}\right), 0\right)-\mathfrak{L} F_{1}(\cdot, \varepsilon)\right\}+F_{1}(t, \varepsilon),
$$

where

$$
\begin{aligned}
& F_{1}(t, \varepsilon)=\varepsilon \int_{a}^{t} \int_{a}^{b} K(\tau, s) Z\left(x_{0}\left(s, c_{r}^{0}\right), s, 0\right) d s d \tau+ \\
& \varepsilon \Psi_{0}(t) D^{+} \int_{a}^{b}\left[A(s) \int_{a}^{s} \int_{a}^{b} K(\tau, s) Z\left(x_{0}\left(s, c_{r}^{0}\right), s, 0\right) d s d \tau+\right. \\
&\left.B(s) \int_{a}^{b} K(s, \tau) Z\left(x_{0}\left(\tau, c_{r}^{0}\right), \tau, 0\right) d \tau\right] d s .
\end{aligned}
$$

In the second stage of the iterative process, we get the following boundary-value problem:

$$
\begin{gathered}
\dot{y}_{2}(t)-\Phi(t) \int_{a}^{b}\left[A(s) y_{2}(s)+B(s) \dot{y}_{2}(s)\right] d s=\varepsilon \int_{a}^{b} K(t, s)\left[Z\left(x_{0}\left(s, c_{r}^{0}\right), s, 0\right)+\right. \\
\left.A_{1}(s)\left[X_{r}(t) c_{1}+\bar{y}_{1}(s, \varepsilon)\right]+R\left(y_{1}(s, \varepsilon), s, \varepsilon\right)\right] d s \\
\mathfrak{L} y_{2}(\cdot)=\varepsilon\left\{J\left(x_{0}\left(\cdot, c_{r}^{0}\right)\right)+l_{1}\left[X_{r}(\cdot) c_{1}+\bar{y}_{1}(\cdot, \varepsilon)\right]+R_{1}\left(y_{1}(\cdot, \varepsilon), \varepsilon\right)\right\}
\end{gathered}
$$

By using the necessary and sufficient conditions for the solvability of this boundaryvalue problem, we arrive at the following algebraic system for 
$c_{1} \in \mathbb{R}^{r}:$

$$
B_{0} c_{1}=\left[\begin{array}{c}
-P_{D_{d_{1}}^{*}} \int_{a}^{b}\left[A(s) \tilde{h}_{2}^{1}(s, \varepsilon)+B(s) h_{2}^{1}(s, \varepsilon)\right] d s \\
-P_{Q_{d_{2}}^{*}}\left\{J\left(x_{0}\left(\cdot, c_{r}^{0}\right)\right)+\mathfrak{L}_{1} \bar{y}_{1}(\cdot, \varepsilon)+\right. \\
\left.R_{1}\left(y_{1}(\cdot, \varepsilon), \cdot, \varepsilon\right)-\mathfrak{L} F_{1}^{2}(\cdot, \varepsilon)\right\},
\end{array}\right]
$$

where

$$
\begin{gathered}
F_{1}^{2}(t, \varepsilon)=\tilde{h}_{2}^{1}(t, \varepsilon)+\Psi_{0}(t) D^{+} \int_{a}^{b}\left[A(s) \tilde{h}_{2}^{1}(s, \varepsilon)+B(s) h_{2}^{1}(s, \varepsilon)\right] d s, \\
h_{2}^{1}(t, \varepsilon)=\int_{a}^{b} K(t, s)\left[A_{1}(s) \bar{y}_{1}(s, \varepsilon)+R\left(y_{1}(s, \varepsilon), s, \varepsilon\right)\right] d s, \tilde{h}_{2}^{1}(t, \varepsilon)=\int_{a}^{t} h_{2}^{1}(s, \varepsilon) d s .
\end{gathered}
$$

Under condition (2.15), this system is solvable. The first approximation $c_{1}$ to $c(\varepsilon)$ has the form

$$
c_{1}=-B_{0}^{+}\left[\begin{array}{c}
-P_{D_{d_{1}}^{*}} \int_{a}^{b}\left[A(s) \tilde{h}_{2}^{1}(s, \varepsilon)+B(s) h_{2}^{1}(s, \varepsilon)\right] d s \\
-P_{Q_{d_{2}}^{*}}\left\{J\left(x_{0}\left(\cdot, c_{r}^{0}\right)\right)+\mathfrak{L}_{1} \bar{y}_{1}(\cdot, \varepsilon)+\right. \\
\left.+R_{1}\left(y_{1}(\cdot, \varepsilon), \cdot, \varepsilon\right)-\mathfrak{L} F_{1}^{2}(\cdot, \varepsilon)\right\}
\end{array}\right] .
$$

For the second approximation $y_{2}(t, \varepsilon)$ to the required $y(t, \varepsilon)$ we get

$$
y_{2}(t, \varepsilon)=X_{r}(t) c_{1}+\bar{y}_{2}(t, \varepsilon) .
$$

Continuing the iterative process, we obtain the following iterative procedure for finding the solution $y(t, \cdot) \in C\left[0, \varepsilon_{0}\right], y(t, 0)=0$ of the boundary-value problem (2.3), (2.4) from the operator system (2.16):

$$
c_{j}=-B_{0}^{+}\left[\begin{array}{c}
y_{j+1}(t, \varepsilon)=X_{r}(t) c_{j}+\bar{y}_{j+1}(t, \varepsilon), \\
-P_{D_{d_{1}}^{*}} \int_{a}^{b}\left[A(s) \tilde{h}_{2}^{j}(s, \varepsilon)+B(s) h_{2}^{j}(s, \varepsilon)\right] d s \\
-P_{Q_{d_{2}}^{*}}\left\{J\left(x_{0}\left(\cdot, c_{r}^{0}\right)\right)+\mathfrak{L}_{1} \bar{y}_{j}(\cdot, \varepsilon)+\right. \\
\left.R_{1}\left(y_{j}(\cdot, \varepsilon), \cdot, \varepsilon\right)-\mathfrak{L} F_{j}^{2}(\cdot, \varepsilon)\right\}
\end{array}\right],
$$




$$
\begin{aligned}
\bar{y}_{j+1}(t, \varepsilon)=\varepsilon \Psi_{0}(t) P_{D_{r_{1}}} Q^{+}\left\{J\left(x_{0}\left(\cdot, c_{r}^{0}\right)\right)+\mathfrak{L}_{1} y_{j}(\cdot, \varepsilon)+\right. \\
\left.R_{1}\left(y_{j}(\cdot, \varepsilon), \varepsilon\right)-\mathfrak{L} F_{j}(\cdot, \varepsilon)\right\}+F_{j}(t, \varepsilon),
\end{aligned}
$$

where

$$
\begin{gathered}
F_{j}(t, \varepsilon)=\tilde{h}_{j}(t, \varepsilon)+\Psi_{0}(t) D^{+} \int_{a}^{b}\left[A(s) \tilde{h}_{j}(s, \varepsilon)+B(s) h_{j}(s, \varepsilon)\right] d s \\
=F_{0}^{1}(t)+F_{j}^{2}(t), \\
F_{0}^{1}(t)=\tilde{h}_{1}(t)+\Psi_{0}(t) D^{+} \int_{a}^{b}\left[A(s) \tilde{h}_{1}(s)+B(s) h_{1}(s)\right] d s, \\
F_{j}^{2}(t, \varepsilon)=\tilde{h}_{2}^{j}(t, \varepsilon)+\Psi_{0}(t) D^{+} \int_{a}^{b}\left[A(s) \tilde{h}_{2}^{j}(s, \varepsilon)+B(s) h_{2}^{j}(s, \varepsilon)\right] d s, \\
h_{j}(t, \varepsilon)=\varepsilon\left(h_{1}(t)+h_{2}^{j}(t, \varepsilon)\right), h_{1}(t)=\int_{a}^{b} K(t, s) A_{1}(s) X_{r}(s) d s, \\
h_{2}^{j}(t, \varepsilon)=\int_{a}^{b} K(t, s)\left[A_{1}(s) \bar{y}_{j-1}(s, \varepsilon)+R\left(y_{j-1}(s, \varepsilon), s, \varepsilon\right)\right] d s, \\
\tilde{h}_{j}(t, \varepsilon)=\int_{a}^{t} h_{j}(s, \varepsilon) d s, \tilde{h}_{1}(t)=\int_{a}^{t} h_{1}(s) d s, \tilde{h}_{2}^{j}(t, \varepsilon)=\int_{a}^{j} h_{2}^{j}(s, \varepsilon) d s .
\end{gathered}
$$

Thus, we have proved the following theorem:

Theorem 3 (The sufficient condition). Assume that, under conditions (2.1), (2.2), the generating boundary-value problem (1.8), (1.9) possesses an r-parameter family of solutions (1.11) $(r=m+n-\operatorname{rank} D-\operatorname{rank} Q)$. Then, under condition (2.15), for each real value of the vector $c_{r}=c_{r}^{0} \in \mathbb{R}^{r}$ satisfying the system of equations (2.3), (2.4) for generating constants, the weakly nonlinear boundary-value problem (1.1)-(1.3) has at least one solution $x=x(t, \varepsilon)$ :

$$
x(\cdot, \varepsilon) \in D_{2}\left([a, b] \backslash\left\{\tau_{i}\right\}_{I}\right), \dot{x}(\cdot, \varepsilon) \in L_{2}[a, b], x(t, \cdot) \in C\left[0, \varepsilon_{0}\right] .
$$

For $\varepsilon=0$ this solution turns into a generating solution $x_{0}\left(t, c_{r}^{0}\right)(1.11)$ and is determined by using the convergent iterative process (2.23) and the relation $x_{j}(t, \varepsilon)=$ $x_{0}\left(t, c_{r}^{0}\right)+y_{j}(t, \varepsilon),(j=0,1,2, \ldots)$. 


\subsection{Relationship between necessary and sufficient conditions.}

By analogy with [7], one can establish the relationship between the $d_{1}+d_{2} \times r$ -dimensional matrix $B_{0}$ used to determine the sufficient condition for the existence of the solution of problem (1.1)-(1.3) and the necessary condition for the existence of this solution, which can be formulated as the existence of a root of the equations for generating constants (2.3), (2.4). The following assertion is true:

Theorem 4. In order that a weakly nonlinear boundary-value problem for the system of integrodifferential equations (1.1)-(1.3) have the solution $x=x(t, \varepsilon)$ :

$$
x(\cdot, \varepsilon) \in D_{2}[a, b], \dot{x}(\cdot, \varepsilon) \in L_{2}[a, b], x(t, \cdot) \in C\left[0, \varepsilon_{0}\right], x(t, 0)=x_{0}\left(t, c_{r}^{0}\right),
$$

where $x_{0}\left(t, c_{r}^{0}\right)$ is the generating solution (1.11) with a constant $c_{r}=c_{r}^{0} \in \mathbb{R}^{r}(r=$ $r_{1}-\operatorname{rank} Q, r_{1}=m+n-\operatorname{rank} D$,) it is necessary that the constant $c_{r}^{0}$ be a real root of the equation for generating constants (2.3), (2.4) and sufficient that the following condition be satisfied:

$$
\left.\operatorname{rank}\left\{B_{0}:=\frac{\partial \bar{F}\left(c_{r}\right)}{\partial c_{r}}\right\}\right|_{c_{r}=c_{r}^{0}}=d_{1}+d_{2} .
$$

Moreover, if $p=r_{1}$, then the last condition means that $c_{r}=c_{r}^{0} \in \mathbb{R}^{r}$ is a simple root of the equation for generating constants (2.3), (2.4).

\section{ACKNOWLEDGEMENT}

The authors thank Professor Alexander Boichuk for their useful advices for the improvement of this note. They also thank the anonymous referee for his/her useful comments helped to improve the presentation.

The Project supported by Grant of NAS of Ukraine for young scientists (20152016).

\section{REFERENCES}

[1] N. Azbelev, V. Maksimov, and L. Rakhmatullina, "Introduction to the theory of functional differential equations: Methods and applications," Contemporary Mathematics and its Applications, vol. 3, pp. 1-318, 2007.

[2] A. Boichuk, M. Langerova, and J. Skorirova, "Bounded solutions of impulsive differential systems," Functional Differential Equations, vol. 18, no. 1-2, pp. p-89, 2011.

[3] A. A. Boichuk, "Boundary-value problems for impulse differential systems," Zeszyty Naukowe, Uniwersytet Jagiellonskiego, vol. 1223, pp. 187-192, 1998.

[4] A. A. Boichuk, "A condition for the existence of a unique Green-Samoilenko function for the problem of invariant torus," Ukrainian Mathematical Journal, vol. 53, no. 4, pp. 637-641, 2001.

[5] A. A. Boichuk, N. A. Perestyuk, and A. M. Samoilenko, "Periodic solutions of impulse differential systems in critical cases," Differential Equations, vol. 27, no. 9, pp. 1070-1073, 1991.

[6] A. A. Boichuk and A. M. Samoilenko, Generalized inverse operators and Fredholm boundaryvalue problems. Utrecht-Boston: Walter de Gruyter, 2004. 
[7] O. Boichuk and I. Holovatska, "Boundary-value problems for systems of integrodifferential equations," Journal of Mathematical Sciences (Translation of Nonlinear Oscillations (Neliniini Kolyvannya), 16 (2013), No. 4), vol. 3, no. 203, pp. 306-321, 2014.

[8] O. A. Boichuk and I. A. Golovatska, "Weakly nonlinear systems of integrodifferential equations," Journal of mathematical sciences (Translation of Nonlinear Oscillations (Neliniini Kolyvannya), 16 (2013), No. 4), vol. 3, no. 201, pp. 288-295, 2014.

[9] I. Bondar, "Weakly perturbed boundary-value problems for systems of integro-differential equations with impulsive action," Tatra Mountains Mathematical Publications, vol. 63, no. 1, pp. 7387,2015

[10] I. Bondar, "Boundary value problems for linear systems of integro-differential equations with impulsive action,” Bukovinian Mathematical Journal, vol. 2, no. 4, pp. 7-11, 2016.

[11] M. I. Gromyak, "Construction of periodic solutions of second-order differential and integrodifferential wave equations," Ukrainian Mathematical Journal, vol. 40, no. 1, pp. 40-44, 1988.

[12] I. A. Holovats'ka, "Weakly perturbed systems of linear integro-differential equations," Journal of Mathematical Sciences (United States), vol. 189, no. 5, pp. 735-750, 2013.

[13] M. A. Krasnoselskii, G. M. Vainikko, and P. P. Zabreiko, Approximate Solution of Operator Equations. Moscow: Nauka, 1968.

[14] I. G. Malkin, Some Problems in the Theory of Nonlinear Oscillations. Moscow: Gostekhizdat, 1956.

[15] A. M. Samoilenko, A. A. Boichuk, and A. A. Boichuk, "Solutions of weakly-perturbed linear systems bounded on the entire axis," Ukrainian Mathematical Journal, vol. 54, no. 11, pp. 1842 $1858,2002$.

[16] A. M. Samoilenko, O. A. Boichuk, and S. A. Krivosheya, "Boundary-value problems for systems of integro-differential equations with degenerate kernel," Ukrainian Mathematical Journal, vol. 48, no. 11, pp. 1785-1789, 1996.

[17] A. Zettl, "Adjoint and self-adjoint BVP's with interface conditions," SIAM J.Appl.Math., vol. 16, no. $4,1968$.

\section{Authors' addresses}

\section{Bondar}

Institute of Mathematics, The National Academy of Science of Ukraine, 3 Tereshchenkivs'ka Str., 01601 Kyiv, UKRAINE

E-mail address: holovatska.ivegmail.com

\section{Gromyak}

Department of Physics and Mathematics, Volodymyr Hnatiuk National Pedagogical University,

10 Vynnychenka Str., 46018 Ternopil, UKRAINE

E-mail address: ghromjakegmail.com

\section{N. Kozlova}

Mechanics and Mathematics Faculty, Taras Shevchenko National University, 4 Akademika Glushkova Avenue, 03127 Kyiv, UKRAINE

E-mail address: nkozlovaa@gmail.com 